\title{
REA_05 - Development of a R\$1 molecular test for rapid and direct detection of chikungunya virus from patient and mosquito samples
}

Severino Jefferson Ribeiro da Silva ${ }^{1 *}$; Jurandy Júnior Ferraz de Magalhães ${ }^{1}$; Renata Pessôa Germano Mendes $^{1}$; Caroline Targino Alves da Silva ${ }^{1}$; Diego Guerra de Albuquerque Cabral ${ }^{2}$; Jacilane Bezerra da Silva $^{2}$; Keith Pardee ${ }^{3}$; Lindomar Pena ${ }^{1}$.

${ }^{1}$ Fiocruz - Fundação Oswaldo Cruz;

${ }^{2}$ LACEN-PE;

${ }^{3}$ University of Toronto.

Introduction: The epidemic of Chikungunya virus (CHIKV) in the Americas has transformed a previously obscure mosquito-borne virus into a global public health concern. In regions where there is a simultaneous circulation of other arboviruses, such as dengue virus (DENV) and Zika virus (ZIKV), the clinical diagnosis of CHIKV infection becomes extremely difficult due to the similarities between their clinical manifestations and sometimes overlapping symptoms. Therefore, laboratory diagnosis is critical to correctly identify the etiological agent. Currently, the reverse transcriptase reaction followed by quantitative polymerase chain reaction (RT-qPCR) is the gold standard for molecular diagnostic of CHIKV in patient and mosquito samples. However, the technique presents several drawbacks, which limits the application for Point-of-care (POC) diagnostics, particularly in low and middle-income countries.

Objective: In this context, the aim of this work was to develop and validate a rapid molecular test based on the reverse transcriptase technique followed by isothermal loop-mediated amplification (RT-LAMP) for detection of CHIKV in patient and mosquito samples.

Methodology: In all experiments, the CHIKV strain PE2016-480 was used. Initially, all reagent concentration as well as all RT-LAMP assay conditions were optimized and established. With the diagnostic assay ready for field testing, it was determined the ability of RT-LAMP to detect CHIKV in human biological and mosquito samples, including serum, urine, saliva and homogenate of Aedes aegypti under controlled conditions. Then, the analytical specificity was evaluated against a panel of different mosquito-borne viruses including DENV (1-4), YFV, ZIKV and MAYV. The analytical sensitivity of the assay was evaluated using a serial dilution of CHIKV. Finally, the proof-of-concept of the RT-LAMP assay was performed with 44 serum samples from patients and the value per reaction was calculated based on the cost of all necessary reagents. In parallel, all samples were tested using the RT-qPCR method as a gold-standard comparison.

Results: Based on the results, the RT-LAMP assay was highly specific for the detection of CHIKV in just 30 minutes and similar sensitivity when compared RT-qPCR for detection of CHIKV. Importantly, the assay reported here is able to detect CHIKV in human and mosquito samples without RNA extraction or expensive equipment. Using 44 patient samples, we find similar diagnostic performance when the platform was compared to the reference test to diagnose CHIKV. As for the cost of each reaction of the RT-LAMP, the value was one Real (R\$1.00).

Conclusion: The CHIKV RT-LAMP assay described here represents a potential alternative and inexpensive POC tool for the molecular diagnosis and routine screening of CHIKV-infection. The test is rapid, simple and robust method for CHIKV detection in patient and mosquito samples with performance equal to RTqPCR. Our POC tool have a great potential for producing reliable results to assist clinicians and can bring decentralization of diagnostic.

Keywords: CHIKV; Diagnostic; Point-of-care 